\title{
An Exploratory Survey of Deqi Sensation from the Views and Experiences of Chinese Patients and Acupuncturists
}

\author{
Hong-Wen Yuan, ${ }^{1,2}$ Liang-Xiao Ma, ${ }^{1,3}$ Peng Zhang, ${ }^{1}$ Chi Lin, ${ }^{1}$ Dan-Dan Qi, ${ }^{1}$ \\ Jing Li, ${ }^{1}$ Si-Yuan Xin, ${ }^{1}$ Ni-Juan Hu, ${ }^{1}$ Chun-Hua Li, ${ }^{4}$ Yu-Qi Liu, ${ }^{5}$ Jie Hao, ${ }^{1}$ Jie-Ping Xie, ${ }^{1}$ \\ Hai Cui, ${ }^{2}$ and Jiang $\mathrm{Zhu}^{1,3}$ \\ ${ }^{1}$ School of Acupuncture Moxibustion and Tuina, Beijing University of Chinese Medicine, Beijing 100029, China \\ ${ }^{2}$ School of Traditional Chinese Medicine, Capital Medical University, Beijing 100069, China \\ ${ }^{3}$ The Key Unit of Evaluation of Characteristic Acupuncture Therapy, State Administration of Traditional Chinese Medicine, \\ Beijing 100029, China \\ ${ }^{4}$ Beijing Electric Power Hospital, Capital Medical University, Beijing 100073, China \\ ${ }^{5}$ Institute of Basic Research in Clinical Medicine, China Academy of Chinese Medical Science, Beijing 100700, China
}

Correspondence should be addressed to Jiang Zhu; jzhjzh@263.net

Received 12 May 2013; Accepted 22 October 2013

Academic Editor: Cun-Zhi Liu

Copyright (C) 2013 Hong-Wen Yuan et al. This is an open access article distributed under the Creative Commons Attribution License, which permits unrestricted use, distribution, and reproduction in any medium, provided the original work is properly cited.

\begin{abstract}
Deqi sensation is believed to be important in clinical efficacy according to TCM theory. The measuring method of Deqi sensation has significant implications for the result of research trials. This study makes an investigation on acupuncture-experienced patients and expert acupuncturists in China and aims to find out the patient's needling sensations and acupuncturist's sensations which can be acceptable as descriptors of Deqi sensation, so as to provide foundation for more systematic and sensitive quantitative evaluation method of Deqi sensation. Results of this survey indicated that the Deqi sensation noted by both patient and acupuncturist is equally important to the treatment efficacy. It is found that there are some differences between the patients' real-life experience and the acupuncturists' expectations on patients' Deqi sensation. The "dull pain," "aching," "sore," "numb," "distended," "heavy," "electric," "throbbing," "warmness," "coolness," "spreading," and "radiating" can be considered as the main manifestations of Deqi sensations. The acupuncturists believed that Deqi sensations were mainly "pulling," "tight," and "throbbing." We suggest developing a questionnaire measuring the Deqi sensations which includes both the sensations of the patient and acupuncturist, and this would be very important and necessary for a better understanding of the relationship between Deqi sensation and acupuncture effects in future studies.
\end{abstract}

\section{Introduction}

Deqi, which first appeared in the "Huang Di Nei Jing," refers to the reaction of movement of qi (vital energy) of the human body when acupuncture needles are inserted into acupoints. It is believed that Deqi was closely related to the treatment efficacy. In clinic, Deqi is mainly testified by needle sensation, which includes the patients' sensations and the acupuncturists' sensations. The patient's needle sensations mainly include sour, numb, swelling, heavy, pain, hot, cold, and the complex feeling and spreading based on these feelings; when the patients have those needling sensations, the acupuncturist can also feel heavy, tense, or needle stuck, or needle tremor [1-3]. The central mechanism of Deqi has been partly explored in some studies [4-9], but it does not have a well understanding. Preliminary findings suggest that Deqi may be an important factor of acupuncture analgesia [10-12]. Measuring the patients' Deqi sensation by using the international scale was originated from the end of the 1980s [13]. In recent years, in order to effectively improve the quality of acupuncture research on Deqi, quantitative measurement of Deqi attracts increasing attention. The representative scales include the MASS scale compiled by Kong et al. (the MGH Acupuncture Sensation Scale) [14] and the Southampton 
Needle Sensation Questionnaire written by White et al. [15]. In addition, some investigators developed scales in Korean [16]. Yu et al. have found that the Chinese version of MASS has good repeatability and internal consistency [17]. However, a standardized, valid, and reliable scale for quantitatively measuring the "Deqi" is still lacking.

In traditional acupuncture, the acupuncturists must pay attention to eliciting and controlling of Deqi. However, modern evaluation tools for Deqi and its related studies all ignore the acupuncturists' sensations. By taking a systematic investigation of the ancient and modern literature, we found that the current evaluation tools can be further improved based on well understanding of Deqi's connotation. In order to develop a validated method to measure the Deqi, we performed a descriptive survey with the following three specific aims: (1) understanding Chinese patients' and acupuncturists' attitudes and beliefs on acupuncture sensations and Deqi, (2) exploring the influence factors of Deqi and the effect of Deqi on acupuncture treatment efficacy, (3) summarizing the patients' Deqi sensations reported by patients and senior acupuncturists, and (4) investigating the acupuncturist's perceptions on Deqi.

In China, the health care system includes both modern medicine and TCM. As acupuncture is very popular in China, Deqi sensations in an acupuncture treatment session are widely accepted by the majority of Chinese patients. Some patients even require obtaining the needling sensations or some other special acupuncture feelings on their own initiative [18]. The patients' attitude towards Deqi sensation was significant different between Chinese patients and those from the US [19]. So in this study, we investigated individual patients' experiences in an actual clinical setting and then consulted expert acupuncturists who have rich clinical experience. The survey is made by observing a homogeneous population undergoing the same style of TCM.

\section{Methods}

2.1. Survey Development. Prior to the formal investigation, a structured interview was setup through a literature review involving both ancient and modern, Chinese and English, and then the terms frequently used for describing the patients' and acupuncturists' perception on Deqi were collected. The Chinese literature includes the ancient books, modern acupuncture text books, and the published articles related to Deqi. The English literature includes the questionnaires of Deqi, or needle sensation, and scales used for measuring Deqi $[13-16,20,21]$ and its related investigations $[19,22$, 23]. The terms in the lists from the English literature were translated by two translators, respectively. We unified the terms that own the same meaning in Chinese but with different expressions in English, such as the terms "stretch," "expansion," "distention," and "fullness," usually appearing in the English literature, are all translated into "Zhang" (fullness/distention) in Chinese, and then we used the item "distention" in English in this paper.

The draft questionnaires were developed based on the interview with four doctoral candidates and four experts with senior professional titles from the School of Acupuncture-Moxibustion and Tuina, Beijing University of Chinese Medicine. These experts and doctoral candidates have rich experience in administrating acupuncture and being treated with acupuncture. Then, two expert acupuncturists and five patients completed the draft questionnaires. We also interviewed them regarding their opinions on using the questionnaires and modified the draft according to their comments. The major modifications for patient's questionnaire include making a detailed and more clear explanation to distinguish the similar items such as "pulling" and "tight," "spreading" and "radiating." In the options of the question "which terms can describe the Deqi sensations of the patients?" some additional interpretations have been added for the terms that may be puzzled to common patients. For example, "dull pain" is interpreted as "mild, moderate or minor pain without a strong inimical feeling," "sharp pain" is interpreted as "a severe pain with a sense of injury," "twinge" as "an unbearable pain with the highest intensity," "pain" as "a pain unable to clarify its nature." In the questionnaire for the acupuncturists, the questions of "spreading distance" and "the direction of spreading" of needling sensation were added.

Finally, the second presurvey was conducted through the interview on another six acupuncture-experienced undergraduates who were not majored in medicine. No modification was made in the first interview. After one week, we altered the order of the questions and conducted the interview for the same subjects again. The results were consistent with those obtained in the first interview.

2.2. Survey Content. There are two types of questionnaires, one is for the patients and the other is for the expert acupuncturists, which are completed by the patients and the experts, respectively.

The patient's questionnaire contained two parts and one open-ended question. Part 1 includes four single choice questions on the patients' perception of Deqi. Part 2 includes questions about the patient's sensations experienced during acupuncture administration. There are two phases in part 2. Phase 1 involves questions mainly asking about the patients' needling sensations. Twenty-six terms are separated into four categories: pain, temperature, sensation spreading, and others. One supplementary blank was at the end for subjects to describe sensations in their own words. Phase 2 includes three questions asking about the patients' emotions during acupuncture. An open-ended question is added at the end, "What is your impression on Deqi?"

There are also two parts and one open-ended question in the questionnaire for the experts. It includes some questions in the questionnaire for the patients in order to get experts' perceptions on Deqi sensation of patients based on their clinical experience. Part 1 includes five multiple choice questions and three single choice questions regarding the understanding of Deqi by the experts. The questions in part 2 aimed to inquire expert's perceptions on needling sensations of the patient (the same questions in patient's questionnaire) and sensations felt by expert's needling hand. An open-ended question is added at the end, "What are your opinions on this questionnaire of Deqi?" 
2.3. Administration. As the Deqi occurrence rate in Chinese clinical trials is $100 \%$ [24], it is hard to calculate the sample of the subjects. According to the suggestions of the experts, we performed a survey to 40 acupuncturists with senior professional titles and 40 patients, respectively. The survey was conducted in the Beijing TCM Hospital affiliated to Capital Medical University, Huguosi TCM Hospital, and Dongzhimen Hospital affiliated to Beijing University of Chinese Medicine in April 2012. All patients could complete the questionnaire by themselves. All questionnaires were completed by those patients and acupuncturists independently. All investigators have been trained before the investigation. The acupuncturists and patients were reminded that this questionnaire is related to the Deqi sensation only deriving from manual acupuncture with filiform needles (as there are varieties of needles in Chinese clinical treatments). The investigators tried to not disturb the subjects during completing the questionnaires and answered any questions of the subjects in a unified way. Only when the investigators found some missed questions or apparently contradictory options, they would take some necessary reminding.

2.4. Statistical Analysis. The questionnaire data was independently collected by two persons. We made a statistics after verification and amending. Descriptive statistics were performed by using the computer and presented by percent (\%).

When inputting the data, we combined some terms which describe similar sensations, such as "warm" and "burnt," considered to be a different degree of "warm," are merged into "warm," "cool" and "cold" are merged in to "cool."

Types of patients' needling sensations perceived by patients and understood by acupuncturists were compared using chi-square analysis. Chi-square analysis and Fisher's exact test (when appropriate) were then performed to explore the effect of expressing by patients and acupuncturists on types of patients' acupuncture sensations. A level of $P<$ 0.05 was determined to be of statistical significance, and all analyses were two-sided. Statistical analysis was performed using SPSS Statistics 17.0.

\section{Results}

3.1. Participants. A total of 40 patients participated in this survey; $70 \%$ of them were men, with an average age of 60.2 years old. Each of them had prior acupuncture experience, and $83 \%$ of the respondents had more than 10 times acupuncture treatment experiences. When needles are insert, $58 \%$ of the patients stated that they achieved needle sensations in each treatment, while the others always (22\%) or occasionally (20\%) have such feelings, and nobody stated that they had never had such feeling. Forty acupuncturists participated in this survey; $65 \%$ were women, with the average age of 48.5 and working time of 20.7 years.

3.2. The Patients' Attitudes and Beliefs towards Deqi. In this study, nearly $70 \%$ of the patients never heard of "Deqi." Among the patients who know about Deqi, four thought
TABLE 1: Patients' attitudes and beliefs about acupuncture sensation (Deqi).

\begin{tabular}{lcc}
\hline Questions & $\begin{array}{c}\text { Attitudes and } \\
\text { beliefs }\end{array}$ & Number/\% \\
\hline Have you heard of Deqi? & Have heard & $12 / 30 \%$ \\
& Never & $28 / 70 \%$ \\
\hline Do you expect to have needling & Expected & $34 / 85 \%$ \\
sensation during acupuncture & Dispensable & $5 / 12.5 \%$ \\
administration? & Try to avoid & $1 / 2.5 \%$ \\
& Hate & $0 / 0 \%$ \\
\hline & Every time & $23 / 57.5 \%$ \\
Have your acupuncturists asked you & Often & $16 / 40 \%$ \\
about your needling sensations? & Seldom & $1 / 2.5 \%$ \\
& Never & $0 / 0 \%$ \\
\hline Do you think needling sensation is & Yes & $36 / 90 \%$ \\
close to the acupuncture treatment & No & $0 / 0 \%$ \\
efficacy? & Not sure & $4 / 10 \%$ \\
\hline
\end{tabular}

that Deqi is a sensation during acupuncture, one thought that Deqi meant the channels were unblocked, and three of them cannot explain the specific meaning. $85 \%$ of the patients expected to have the needle sensations upon the treatment, and only one did not expect to have needling sensation. About $90 \%$ of the patients thought needling sensation is closely related to the treatment efficacy, and others did not know their relationship. About $58 \%$ of the respondents said that the acupuncturist will ask them about their needle sensations during every acupuncture treatment session. Nearly $40 \%$ of the patients said that they were always asked by their acupuncturists. The patients in this study all have been asked about their needling sensation by their acupuncturists during the treatment, as shown in Table 1.

3.3. The Acupuncturists' Understanding about Deqi. In this study, $95 \%$ of the acupuncturists in China thought that "Deqi" should be elicited in the treatment, while others thought the treatment without needling sensation can also achieve the efficacy. Some experts said that needling sensation could not be elicited in every point due to limitation of treatment time. They said that the patients expected to get a stronger needling sensation, which is inconsistent with the results concluded from the questionnaires for the patients.

Most acupuncturists (90\%) held that acupuncturists sensations felt by their needling hand and the needling sensations of the patients are the typical characteristics of Deqi. Compared to the acupuncturists, the patients are more easily to get needling sensation. In this study, all of the acupuncturists believed that the sensations of the acupuncturists can be a sign of Deqi.

About $73 \%$ of acupuncturists thought that Deqi sensation is related to the treatment efficacy, but only $38 \%$ of them thought that the symptoms can be relieved immediately. Most acupuncturists believed that the patients' sense of Deqi and the acupuncturists sensations are equally important to the treatment efficacy, as shown in Table 2. 
TABLE 2: Acupuncturists' understanding about Deqi.

\begin{tabular}{|c|c|c|}
\hline Questions & Understanding & Number/\% \\
\hline \multirow{3}{*}{$\begin{array}{l}\text { Do you expect to get Deqi } \\
\text { sensation in the } \\
\text { acupuncture treatment? }\end{array}$} & Yes & $38 / 95 \%$ \\
\hline & No & $1 / 2.5 \%$ \\
\hline & Do not care & $1 / 2.5 \%$ \\
\hline \multirow{5}{*}{$\begin{array}{l}\text { Do your patients expect to } \\
\text { get Deqi sensation in the } \\
\text { acupuncture treatment? }\end{array}$} & Expected & $29 / 72.5 \%$ \\
\hline & Dispensable & $9 / 22.5 \%$ \\
\hline & Try to avoid & $0 / 0 \%$ \\
\hline & Very fear & $0 / 0 \%$ \\
\hline & Not sure & $1 / 2.5 \%$ \\
\hline \multirow{4}{*}{$\begin{array}{l}\text { What do you think are the } \\
\text { signs of Deqi? }\end{array}$} & $\begin{array}{l}\text { Patients needle } \\
\text { sensation }\end{array}$ & $36 / 90 \%$ \\
\hline & $\begin{array}{l}\text { Acupuncturists } \\
\text { experiencing } \\
\text { sensations }\end{array}$ & $39 / 97.5 \%$ \\
\hline & Muscle throbbing & $20 / 50 \%$ \\
\hline & Immediate relief & $15 / 37.5 \%$ \\
\hline \multirow{3}{*}{$\begin{array}{l}\text { Which are more likely to } \\
\text { occur, the patients needle } \\
\text { sensations or the } \\
\text { acupuncturists sensations? }\end{array}$} & $\begin{array}{l}\text { Acupuncturists } \\
\text { experiencing } \\
\text { sensations }\end{array}$ & $11 / 27.5 \%$ \\
\hline & $\begin{array}{l}\text { Patients needle } \\
\text { sensations }\end{array}$ & $20 / 50 \%$ \\
\hline & At the same time & $9 / 22.5 \%$ \\
\hline \multirow{3}{*}{$\begin{array}{l}\text { Do you think the Deqi } \\
\text { sensation is related to the } \\
\text { clinical efficacy? }\end{array}$} & Has & $29 / 72.5 \%$ \\
\hline & No & $1 / 2.5 \%$ \\
\hline & Not sure & $10 / 25 \%$ \\
\hline \multirow{4}{*}{$\begin{array}{l}\text { Between the acupuncturists } \\
\text { sensations and the patients } \\
\text { needle sensation, which do } \\
\text { you think is more relevant } \\
\text { to its efficacy? }\end{array}$} & $\begin{array}{l}\text { Acupuncturists } \\
\text { experiencing } \\
\text { sensations }\end{array}$ & $8 / 20 \%$ \\
\hline & $\begin{array}{l}\text { Patients needle } \\
\text { sensations }\end{array}$ & $13 / 35 \%$ \\
\hline & $\begin{array}{l}\text { With same } \\
\text { significance }\end{array}$ & $18 / 45 \%$ \\
\hline & Not related & $1 / 2.5 \%$ \\
\hline
\end{tabular}

Regarding the relationship between Deqi sensation and treatment efficacy, most acupuncturists thought that the efficacy is closely related to the existence of Deqi sensation (87\%), the realization of qi spreading to the affected parts (47\%), and the intensity of Deqi sensation (37\%). In addition, one-third of the acupuncturists thought that the time for inducing Deqi sensation and the direction of sensation spreading may affect the efficacy. Only a few patients thought that the lasting time of Deqi sensation (11\%) and what kind of sensation (8\%) may affect the efficacy.

In addition, the acupuncturists held that the acupoint location (87.5\%), point selection (82.5\%), and the acupuncture manipulation $(90 \%)$ are the three key elements in acupuncture. The physiques of the patients (80\%), acupuncturist's needling skill (67.5\%), needle depth (60\%), the thickness of needle (57.5\%), the type of disease (45\%), the patient's emotional and psychological states (40\%), acupoints selection $(35 \%)$, needle inserting site $(35 \%)$, needle retention
TABle 3: Types of patients' Deqi sensation.

\begin{tabular}{lccc}
\hline Sensation & $\begin{array}{c}\text { Experienced } \\
\text { by patients } \%\end{array}$ & $\begin{array}{c}\text { Considered by } \\
\text { acupuncturists } \%\end{array}$ & \begin{tabular}{c}
$P$ value \\
\hline Related to pain
\end{tabular} \\
Dull pain & $92.5 \%$ & $97.5 \%$ & 0.608 \\
Shape pain & $47.5 \%$ & $60 \%$ & 0.262 \\
Twinge & $10 \%$ & $2.5 \%$ & 0.356 \\
Deep pain & $5 \%$ & $2.5 \%$ & 1.000 \\
Superficial pain & $17.5 \%$ & $30 \%$ & 1.000 \\
Pricking & $27.5 \%$ & $20 \%$ & 0.284 \\
Throbbing pain & $32.5 \%$ & $7.5 \%$ & 0.005 \\
Aching & $12.5 \%$ & $10 \%$ & 1.000 \\
Pain & $65 \%$ & $85 \%$ & 0.039 \\
Related to temperature & $10 \%$ & 0 & 0.124 \\
Warmness & $25 \%$ & $92.5 \%$ & 0.000 \\
Burning & $45 \%$ & $92.5 \%$ & 0.000 \\
Coolness & $2.5 \%$ & $12.5 \%$ & 0.203 \\
Other feelings & $2.5 \%$ & $47.5 \%$ & 0.000 \\
Penetrating & & & \\
Sore & $17.5 \%$ & $10 \%$ & 0.330 \\
Numb & $60 \%$ & $87.5 \%$ & 0.005 \\
Distended & $47.5 \%$ & $75 \%$ & 0.012 \\
Heavy & $72.5 \%$ & $85 \%$ & 0.172 \\
Pressure & $12.5 \%$ & $77.5 \%$ & 0.000 \\
Pinch & $2.5 \%$ & $30 \%$ & 0.001 \\
Pulling & $2.5 \%$ & $7.5 \%$ & 0.608 \\
Tight & $17.5 \%$ & $37.5 \%$ & 0.045 \\
Electric & $7.5 \%$ & $30 \%$ & 0.010 \\
Formicating & $42.5 \%$ & $50 \%$ & 0.501 \\
Throbbing & $10 \%$ & $22.5 \%$ & 0.130 \\
Related to spreading & $72.5 \%$ & $45 \%$ & 0.491 \\
Rpreading & $60 \%$ & $100 \%$ & 0.000 \\
\hline
\end{tabular}

time $(22.5 \%)$, season while needling $(25 \%)$, and time of acupuncture administration (15\%) may all affect the efficacy.

3.4. The Patients' Perceptions of Deqi Sensation. The most common acupuncture sensations reported as experienced by patients were "distended" (72.5\%), "aching" (65\%), "sore" (60\%), those "spreading" (60\%), and, reported as considered by acupuncturists were "warmness" (92.5\%), "sore" (87.5\%), "aching" (85\%), "distended" (85\%), and "spreading" (82.5\%), as shown in Table 3.

In this survey, $82.5 \%$ of the patients believed that Deqi sensation is not harmful in the treatment. However, nearly half of the patients considered that Deqi sensation is tolerable; none of them felt Deqi cannot be tolerated. Half of the respondents stated that Deqi sensation is comfortable. Some patients indicated that Deqi is just a series of sensations; they do not care whether it is comfortable or not, as shown in Table 4. 
TABle 4: Patients' affections about Deqi sensation.

\begin{tabular}{lcc}
\hline Questions & Affections & Number/\% \\
\hline What do you think of & Harmful & $1 / 2.5 \%$ \\
needle sensation/Deqi in & No harm & $33 / 82.5 \%$ \\
the treatment? & Unknown & $6 / 15 \%$ \\
\hline & Negligible & $4 / 10 \%$ \\
& Gentle & $21 / 52.5 \%$ \\
What do you think of Deqi & Strong & $7 / 17.5 \%$ \\
sensation? & Unbearable & $0 / 0 \%$ \\
& Sometimes strong, & $7 / 17.5 \%$ \\
\hline What is your usual & sometimes weak & \\
perception of Deqi & Comfortable & $20 / 50 \%$ \\
sensation? & Uncomfortable & $0 / 0 \%$ \\
& Does not matter & $20 / 50 \%$ \\
\hline
\end{tabular}

3.5. The Relationship between Patients' Experience and Acupuncturists' Consideration of Deqi Sensation. In this study, we not only asked patients to select the terms on behalf of the needle sensation, but also invited some expert acupuncturists to filter them. It showed that there are no statistical differences among the 14 descriptors of the all 26 descriptors $(P>0.05)$. However, there are some differences between the patients' real-life experience and the acupuncturists' expectations among the others, as shown in Table 3.

As long as the patients or acupuncturists select one option among the feelings in the same category, it will be considered to select this kind of feeling in statistics. After comparison, it is found that there is no significant difference between the patients and acupuncturists in selecting the pain related feeling $(P>0.05)$, which indicated that the majority of patients and acupuncturists believed that Deqi sensation is associated with pain.

There are significant differences between patients and acupuncturists in selecting the temperature feel and feeling spreading $(P=0.000)$, which suggested that patients actually feel the temperature changes and needling sensation spreading occurred less than the acupuncturists' expectation.

There are no differences between the "dull pain," "distended," "electric," and "throbbing" between two groups of subjects $(P>0.05)$. All of these sensations are selected by more than half of the patients and acupuncturists, indicating that the feelings are thought to present signs of Deqi sensations.

Referring to the terms "sharp pain," "twinge," "throbbing pain," "burning," "penetrating," "formicating," their supporting rate has no statistical difference between the two groups $(P>0.05)$. Moreover, all are below $20 \%$, indicating that these feelings mentioned above are not the typical Deqi sensations. One-third of the patients selected "pricking" significantly higher than the selection rate of acupuncturists $(P=0.005)$.

3.6. The Acupuncturists' Experience of Needle Grasping Sensation. The most common sensations reported by acupuncturists were "pulling" (95\%), which was described as "as a fish
TABLE 5: Types of acupuncturists experiencing sensation.

\begin{tabular}{lc}
\hline Sensation & $\begin{array}{c}\text { Experienced by } \\
\text { acupuncturists } \%\end{array}$ \\
\hline Pulling/heavy/like a fish biting the hook & $38 / 95 \%$ \\
Tight & $28 / 70 \%$ \\
Throbbing & $6 / 15 \%$ \\
Opposable & $1 / 2.5 \%$ \\
\hline
\end{tabular}

biting the hook" in ancient books, and "tight" (70\%) as shown in Table 5.

\section{Discussion}

4.1. The Choice of the Respondents. One purpose of this survey is to filter the descriptors of the patients' Deqi sensation and the acupuncturists' sensations, so as to develop proper clinical evaluation questionnaires on Deqi. So it is expected to select acupuncture-experienced subjects, especially those patients who already recognized the Deqi sensation. This study is different from some previous studies which selected naive volunteers without any acupuncture experience as their subjects $[13,14,22]$. It has been reported that acupuncture not only benefits the chronic pain $[25,26]$, but also can be an effective adjunctive treatment for facilitating stroke rehabilitation [27, 28]; therefore, acupuncture is widely used for facilitating stroke rehabilitation in China $[29,30]$.

The long treatment course and large amount of stimulation in this treatment are particularly in consistent with the requirements of this survey. So in this study, we selected the patients from three outpatient acupuncture clinics of Beijing TCM hospitals for the survey. $70 \%$ of the respondents suffered from apoplexy and the others from facial paralysis, trauma, headache, low back pain, and gastrohelcoma. More than $80 \%$ of these patients have received more than 10 sessions of acupuncture treatment. They had more experiences in perceiving Deqi sensation and could rule out the needle sensations by accident. Compared with the respondents in experimental models, a survey based on these respondents may reflect the real phenomena of Deqi sensation in the real world clinical setting.

4.2. The Chinese Patients' and Acupuncturists' Attitudes of Deqi. The majority of the patients thought that needling sensations are related to the treatment efficacy and excepted to experience these sensations in the treatment. Nearly half of the patients thought Deqi is comfortable. Although some patients may have felt uncomfortable sometimes, they also thought it is not harmful. Our result is consistent with Mao's et al. result [31]. Regarding the open-ended questions, two patients said that eliciting the Deqi sensations indicated that the acupuncturist had good needling skills.

In this survey, three quarters of the 40 experts thought Deqi sensation is closely related to the treatment efficacy. Although 25\% of the acupuncturists were not sure about the relationship between Deqi sensation and treatment efficacy, they still excepted to elicit the Deqi sensation in clinical. 
It may be influenced by the acupuncture education and the demands of the patients. Only one acupuncturist thought Deqi sensation is not related to treatment efficacy. So it is obviously shown that the acupuncturists and patients, especially the patients who suffered more severe diseases, are eager to achieve Deqi sensation and highly expected its influence on efficacy.

4.3. The Patients' Deqi Sensation. This survey was a retrospective study, which filtered the terms to describe patient's Deqi sensations based on their memories. $92.5 \%$ of patients reported at least one pain related term. There are three terms used to describe the intensity of pain, "dull pain," "sharp pain," and "twinge." Half of the patients chose dull pain, four of them chose sharp pain and dull pain, and one patient chose dull pain and twinge at the same time as the twinge occasionally occurred and it is difficult to distinguish them. Although most patients cannot distinguish pain and Deqi sensation, "dull pain" is widely accepted to be one of the Deqi sensations. In two terms of "deep pain" and "skin pain" associated with location of pain, more patients chose skin pain, indicating that the patients may have an incorrect understanding of Deqi sensation. Deqi sensation is the needle sensation when the needle was inserted into the acupoints with a certain depth, which pointed out that the pain caused by skin penetrating during needle inserting should be distinguished so as to avoid confusion of the skin pain and Deqi sensation. Among the twenty-six terms for needle sensations in this questionnaire, "fullness" was the most common sensation (72.5\%), followed by the "aching" (65\%) and "sore" (60\%); approximately half of the patients selected "dull pain," "numb," "spreading," which can be considered as the characteristics of Deqi sensations.

In previous studies of filtering Deqi sensation terms, the investigated objects include both patients and acupuncturists $[13,14,20,22,23]$. This survey is quite different from previous studies. We filtered the terms of Deqi sensations by the patients and expert acupuncturists in the same hospital at the same time. After comparing, it is found that there exist some differences between the real-life experiences of patients and acupuncturists' expectation.

It was found that there are no significant differences between the patients (92.5\%) and acupuncturists (97.5\%) in selecting the pain related terms $(P>0.05)$, suggesting that both of them believe that Deqi sensation is associated with pain. There are experimental studies that confirmed that the basic material for generating acupuncture needles sensation is mainly the pain receptors located in the acupoints with a depth $[32,33]$. The dull pain, aching, sore, and distended sensation is consistent with its reflection in brain [34], so it showed that Deqi and pain cannot be completely separated in physiology.

There are much more acupuncturists (92.5\%) selecting the temperature related sensation than the patients $(25 \%)$ $(P=0.000)$. It suggests that patients actually rarely feel the temperature changes during acupuncture than the acupuncturist expected. A lot of heat- or cold-inducing needling manipulations recorded in acupuncture classics may be the reason that most acupuncturists tend to choose the warm feeling as the sign of Deqi (92.5\%). Although the sense of cool is usually mentioned in the literature, but only $47.5 \%$ acupuncturists had successfully elicited a sense of cool and achieved a better efficacy at the same time. (This might be caused by the types of diseases, and the cool sensation is more difficult to be elicited.) As there is only 1 patient who had experienced such feeling, significant differences existed between the two groups $(P=0.000)$. However, we believed that the difference was just due to the small sample in this survey.

Although it has a significant statistical difference $(P=$ $0.000)$ between the patients $(72.5 \%)$ and acupuncturists (100\%) in selecting the sensation spreading $(P=0.000)$, the choosing rates of the two groups of people are relatively high, which means that propagated sensation along channel is also one of the typical manifestations of Deqi sensations.

Among the twenty-six terms, "dull pain," "sore," "aching," "numb," "distended," "electric," "warmness," and "spreading," their supporting rate reached $50 \%$ or above, and these feelings are the common feelings appearing in Deqi sensation. However, the supporting rate of "sharp pain," "twinge," "throbbing pain," "burning," "penetrating," and "formicating" were below $20 \%$, indicating that these are not the typical signs of Deqi sensations.

About $1 / 3$ of the patients chose the "sting" significantly higher than the choice rate of the acupuncturists $(P=0.005)$. The terms "pricking" and "stinging" occurring rate is above $60 \%$ among the young females, which is nearly one time higher than the $32.5 \%$ in this study [22]. It obviously showed that pricking always appeared accompanied with Deqi sensations, but most acupuncturists thought that pricking is only a feeling during needle inserting and does not belong to Deqi sensation [13, 34-37]. Recently, it has been found that pricking pain is not commonly regarded as Deqi sensation, so the acupuncturists should avoid pricking pain to the patients.

The choose rates of "coolness," "heavy," and "radiating" among the acupuncturists are all above 50\%, while they are below 20\% among the patients. The "coolness" discussed above is one type of Deqi sensations as mentioned in ancient books. However, "coolness" is hard to be elicited in the clinical due to the type of disease, which may be the main reason that there was a significant difference between the patients and acupuncturists. In two hundred cases of retrospective investigation, $57.5 \%$ of patients achieved a heavy sense [31], and another two hundred and twenty seven cases in a survey conducted in UK stated that the heavy sense choosing rate is $20 \%$ [15], which confirmed that heavy is a possible sense of Deqi sensation. There are "radiating" related terms in the literature of Vincent et al. [13] and Park et al. [22]. A survey of Korean women with a similar sample size reported that "radiating" appearance rate is 76.3\% [23], while it disappeared in the MASS and SNSQ list. In this study, in order to facilitate patients' selections, we added explanations for the terms radiating and spreading. Radiating: needles sensation spread without fixed directions; spreading: needles sensation spread with a fixed direction.

4.4. The Acupuncturist's Needle Grasping Sensation of Deqi. In recent Deqi studies, more attention is paid to the patients' needle sensations than the acupuncturists sensations [13-16]. 
However, according to traditional theory the acupuncturists' sensations are more important in eliciting and controlling Deqi. Therefore, this survey adds more terms of the description of the acupuncturists' sensations. In this study, most expert acupuncturists believed that Deqi sensation is mainly manifested by "pulling" and "tight", which is the same as described in ancient Chinese medicine. A few of them thought "throbbing" is beneficial to the treatment of cerebrovascular disease caused by motor dysfunction and other diseases, which is regarded as one kind of Deqi sensation. However, most acupuncturists do not agree with "opposable" mentioned in some Chinese pieces of literature (5\%).

4.5. Limits. Some acupuncture sensations had to be translated from English into Chinese, and we have to add certain additional interpretations for better understanding by Chinese patients. Then, all the terms had to be retranslated to English again, whereby the delicate meaning of some of the items may not have been fully conveyed in its Chinese equivalent. The sample size of this study was not big enough, and the scope of this survey is narrow.

All of the objects in this study were acupuncture-experienced patients, and more are men. However, this investigation can still reflect the general recognitions of Deqi sensations among the Chinese patients and acupuncturists. In the future, we would make an investigation with a larger sample and analyze the relation of the frequency of occurrence of different Deqi sensation with curative effect.

\section{Conclusion}

Results of this survey indicated that acupuncturists and patients, especially the patients in serious condition, are eager to get Deqi sensations and highly expected to get better efficacy due to stronger Deqi sensation.

Different from previous studies, this survey filtered the terms of Deqi sensations by the patients and experts in the same hospital at the same time. After comparing, it is found that there exist some differences between the real-life experiences of patients and acupuncturists' expectation. It is found that pain related feeling and sensation spreading feeling are the major manifestations of Deqi sensations. However, the patients actually feel the temperature changes, and acupuncture conduction occurred less than the acupuncturists' expectation.

The "dull pain," “sore," "aching," "numb," “distended," "electric," "throbbing," “warmness," "spreading," “coolness," "heavy," and "radiating" can be considered as the main manifestations of Deqi sensations, while "sharp pain," "twinge," "pricking," "throbbing pain," "burning," "penetrating," "pinch," and "formicating" may not be the typical signs of Deqi sensations. The acupuncturists' Deqi sensations are mainly described as "pulling," "tight," and "throbbing".

\section{Conflict of Interests}

The authors declare that they do not have a direct financial relationship with the commercial identities mentioned in this paper.

\section{Authors' Contribution}

Hong-Wen Yuan and Liang-Xiao Ma contributed equally to this work.

\section{Acknowledgments}

The authors sincerely thank the research staff at Dongzhimen Hospital Affiliated to Beijing University of Chinese Medicine, Beijing Hospital of Traditional Chinese Medicine affiliated to Capital Medical University, and Huguosi Hospital of Traditional Chinese Medicine affiliated to Beijing University of Chinese Medicine. The authors also sincerely thank the National Basic Research Program of China (973 Program) (no. 2012CB518506 and no. 2006CB504503), the National Natural Science Foundation of China (no. 30973793), and the Doctoral Program of Higher Education of Ministry of Education of China (no. 20090013110005) for funding this study.

\section{References}

[1] X. Cheng, Chinese Acupuncture and Moxibustion, Foreign Languages Press, Beijing, China, 1987.

[2] Y. X. Yuan, J. Ren, and L. Huang, Chinese-English Dictionary of Traditional Chinese Medicine, People's Medical Publishing Press, Beijing, China, 1997.

[3] X. M. Shi, Text of Acupuncture and Moxibustion, China Traditional Medicine Press, Beijing, China, 2007.

[4] J. Bossy, D. Prat, and P. Sambuc, "Sensory potentials evoked by stimulation of the Jing points at the hand," Acupuncture and Electro-Therapeutics Research, vol. 9, no. 4, pp. 195-201, 1984.

[5] K. M. Wang, S. M. Yao, Y. L. Xian, and Z. L. Hou, "A study on the receptive field of acupoints and the relationship between characteristics of needling sensation and groups of afferent fibres," Scientia Sinica B, vol. 28, no. 9, pp. 963-971, 1985.

[6] F. Kagitani, S. Uchida, H. Hotta, and Y. Aikawa, "Manual acupuncture needle stimulation of the rat hindlimb activates groups I, II, III and IV single afferent nerve fibers in the dorsal spinal roots," Japanese Journal of Physiology, vol. 55, no. 3, pp. 149-155, 2005.

[7] A. Campbell, "Role of $\mathrm{C}$ tactile fibres in touch and emotionclinical and research relevance to acupuncture," Acupuncture in Medicine, vol. 24, no. 4, pp. 169-171, 2006.

[8] J. Kong, R. L. Gollub, J. M. Webb, J.-T. Kong, M. G. Vangel, and K. Kwong, "Test-retest study of fMRI signal change evoked by electroacupuncture stimulation," NeuroImage, vol. 34, no. 3, pp. 1171-1181, 2007.

[9] Y. Huang, T. J. Zeng, G. F. Zhang et al., "Activated and deactivated functional brain areas in the Deqi state A functional MRI study," Neural Regeneration Research, vol. 7, no. 30, pp. 23622369, 2012.

[10] X. Cao, "Scientific bases of acupuncture analgesia," Acupuncture and Electro-Therapeutics Research, vol. 27, no. 1, pp. 1-14, 2002.

[11] L. Ai, J. P. Dai, B. X. Zhao et al., "Investigation of analgesic mechanism of acupuncture: a fMRI study," Chinese Journal of Medical Imaging Technology, vol. 20, no. 8, pp. 1197-1200, 2004.

[12] G. H. Kim, M. Yeom, C. S. Yin et al., "Acupuncture manipulation enhances anti-nociceptive effect on formalin-induced pain in rats," Neurological Research, vol. 32, no. 1, pp. S92-S95, 2010. 
[13] C. A. Vincent, P. H. Richardson, J. J. Black, and C. E. Pither, "The significance of needle placement site in acupuncture," Journal of Psychosomatic Research, vol. 33, no. 4, pp. 489-496, 1989.

[14] J. Kong, R. Gollub, T. Huang et al., "Acupuncture De Qi, from qualitative history to quantitative measurement," Journal of Alternative and Complementary Medicine, vol. 13, no. 10, pp. 1059-1070, 2007.

[15] P. White, F. Bishop, H. Hardy et al., "Southampton needle sensation questionnaire: development and validation of a measure to gauge acupuncture needle sensation," Journal of Alternative and Complementary Medicine, vol. 14, no. 4, pp. 373-379, 2008.

[16] Y. Kim, J. Park, H. Lee, H. Bang, and H.-J. Park, "Content validity of an acupuncture sensation questionnaire," Journal of Alternative and Complementary Medicine, vol. 14, no. 8, pp. 957$963,2008$.

[17] D. T. Yu, A. Y. Jones, and M. Y. Pang, "Development and validation of the Chinese version of the Massachusetts General Hospital Acupuncture Sensation Scale: an exploratory and methodological study," Acupuncture in Medicine, vol. 30, no. 3, pp. 214-221, 2012.

[18] T. Huang and R. H. Wang, "An investigation report about the present situation of acupuncture Deqi," Medicine \& Philosophy, vol. 31, no. 4, pp. 66-67, 2010.

[19] K. K. K. S. Hui, T. N. Sporko, M. G. Vangel, M. Li, J. Fang, and L. Lao, "Perception of Deqi by Chinese and American acupuncturists: a pilot survey," Chinese Medicine, vol. 6, article 2, 2011.

[20] J. Kong, D. T. Fufa, A. J. Gerber et al., "Psychophysical outcomes from a randomized pilot study of manual, electro, and sham acupuncture treatment on experimentally induced thermal pain," Journal of Pain, vol. 6, no. 1, pp. 55-64, 2005.

[21] C. S. Yin, J. Park, J.-Y. Lee et al., "Acupuncture perception (Deqi) varies over different points and by gender with two distinct distribution patterns of dullness and pain," Journal of Sensory Studies, vol. 24, no. 5, pp. 635-647, 2009.

[22] H. Park, J. Park, H. Lee, and H. Lee, "Does Deqi (needle sensation) exist?" American Journal of Chinese Medicine, vol. 30, no. 1, pp. 45-50, 2002.

[23] J. Park, H. Park, H. Lee, S. Lim, K. Ahn, and H. Lee, “Deqi sensation between the acupuncture-experienced and the Naïve: a Korean study II," American Journal of Chinese Medicine, vol. 33, no. 2, pp. 329-337, 2005.

[24] J. Xiong, Q. Yuan, L. J. Zhang et al., "Preliminary observation of acupuncture Deqi in clinical," Journal of Traditional Chinese Medicine, vol. 51, no. 2, pp. 212-214, 2010.

[25] A. J. Vickers, A. M. Cronin et al., "Acupuncture for chronic pain: individual patient data meta-analysis," Archives of Internal Medicine, vol. 172, no. 19, pp. 1444-1453, 2012.

[26] R. R. Coeytaux and J. J. Park, "Acupuncture research in the era of comparative effectiveness research," Archives of Internal Medicine, vol. 158, no. 4, pp. 287-288, 2013.

[27] "NIH Consensus Statement," 1997.

[28] S. C. Shiflett, "Does acupuncture work for stroke rehabilitation: what do recent clinical trials really show?" Topics in Stroke Rehabilitation, vol. 14, no. 4, pp. 40-58, 2007.

[29] S. Zhang, N. Li, and M. Liu, "Use of acupuncture for stroke in China," Acupuncture in Medicine, vol. 27, no. 4, p. 146, 2009.

[30] X. F. Zhao, Y. Du, P. G. Liu et al., "Acupuncture for stroke: evidence of effectiveness, safety, and cost from systematic reviews," Topics in Stroke Rehabilitation, vol. 19, no. 3, pp. 226233, 2012
[31] J. J. Mao, J. T. Farrar, K. Armstrong, A. Donahue, J. Ngo, and M. A. Bowman, "De qi: Chinese acupuncture patients' experiences and beliefs regarding acupuncture needling sensationan exploratory survey," Acupuncture in Medicine, vol. 25, no. 4, pp. 158-165, 2007.

[32] K. Wang and J. Liu, "Needling sensation receptor of an acupoint supplied by the median nerve-studies of their electrophysiological characteristics," American Journal of Chinese Medicine, vol. 17, no. 3-4, pp. 145-156, 1989.

[33] Q. S. Dong, X. Q. Wang, R. T. Zhang et al., "Discuss the material basis of manual acupuncture from the relationship between manual acupuncture sensation and sensory function in acupoint," Journal of Sichuan of Traditional Chinese Medicine, vol. 25, no. 1, pp. 92-97, 2007.

[34] K. K. S. Hui, E. E. Nixon, M. G. Vangel et al., "Characterization of the Deqi response in acupuncture," BMC Complementary and Alternative Medicine, vol. 7, article 33, 2007.

[35] K. K. S. Hui, O. Marina, J. D. Claunch et al., "Acupuncture mobilizes the brain's default mode and its anti-correlated network in healthy subjects," Brain Research, vol. 1287, pp. 84103, 2009.

[36] K. K. S. Hui, O. Marina, J. Liu, B. R. Rosen, and K. K. Kwong, "Acupuncture, the limbic system, and the anticorrelated networks of the brain," Autonomic Neuroscience, vol. 157, no. 1-2, pp. 81-90, 2010.

[37] A. U. Asghar, G. Green, M. F. Lythgoe, G. Lewith, and H. MacPherson, "Acupuncture needling sensation: the neural correlates of Deqi using fMRI," Brain Research, vol. 1315, pp. 111-118, 2010. 


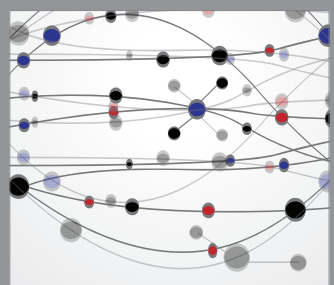

The Scientific World Journal
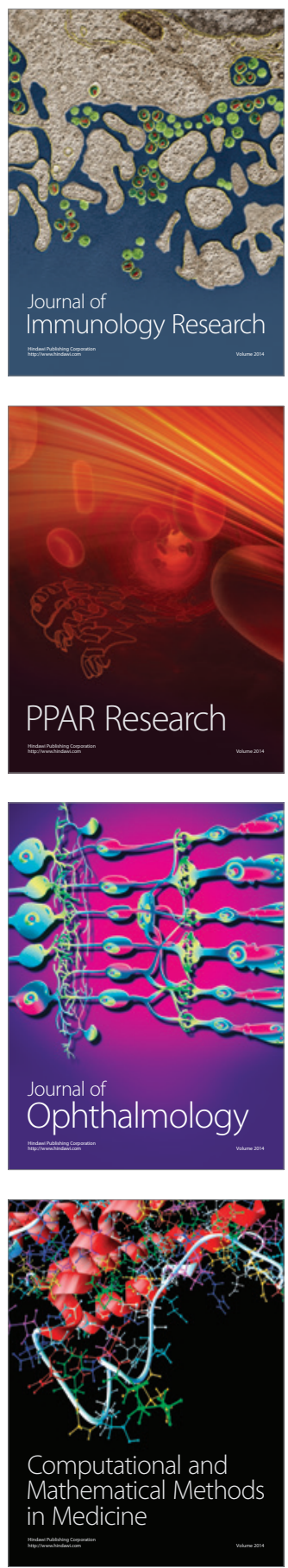

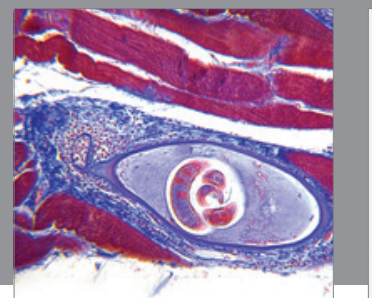

Gastroenterology

Research and Practice
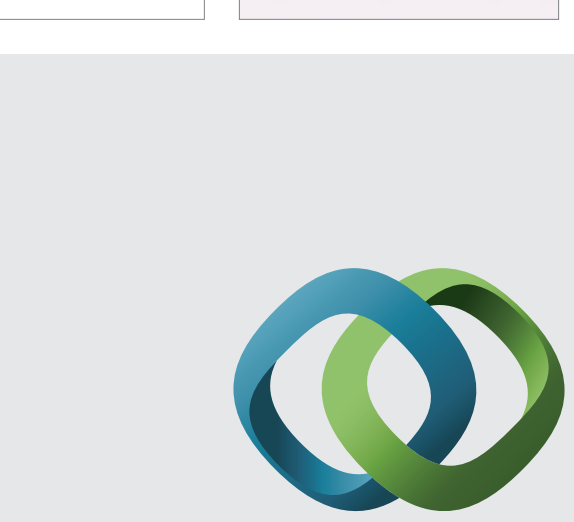

\section{Hindawi}

Submit your manuscripts at

http://www.hindawi.com
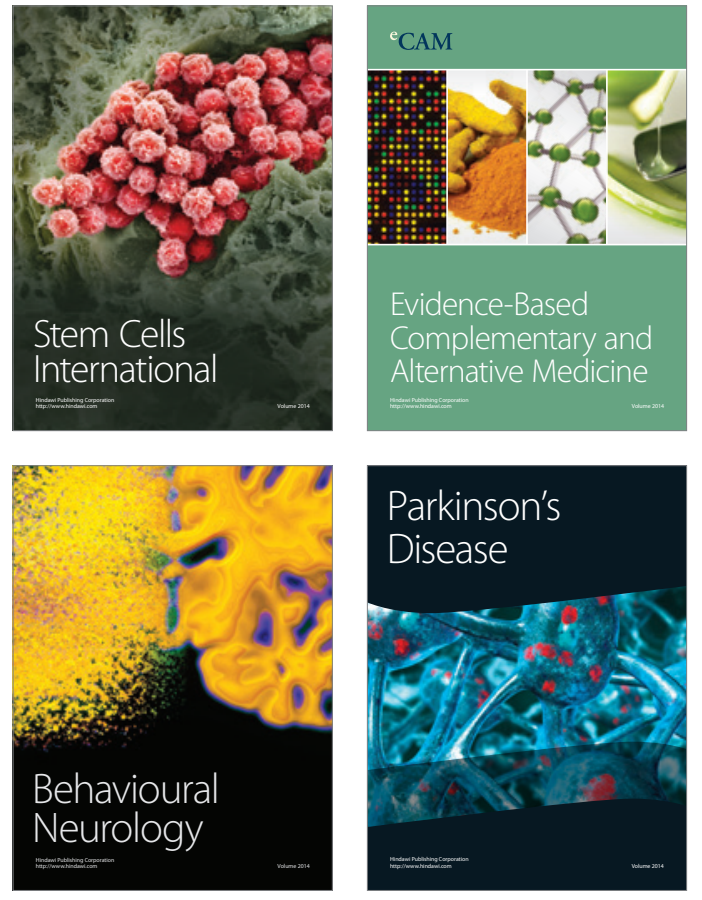
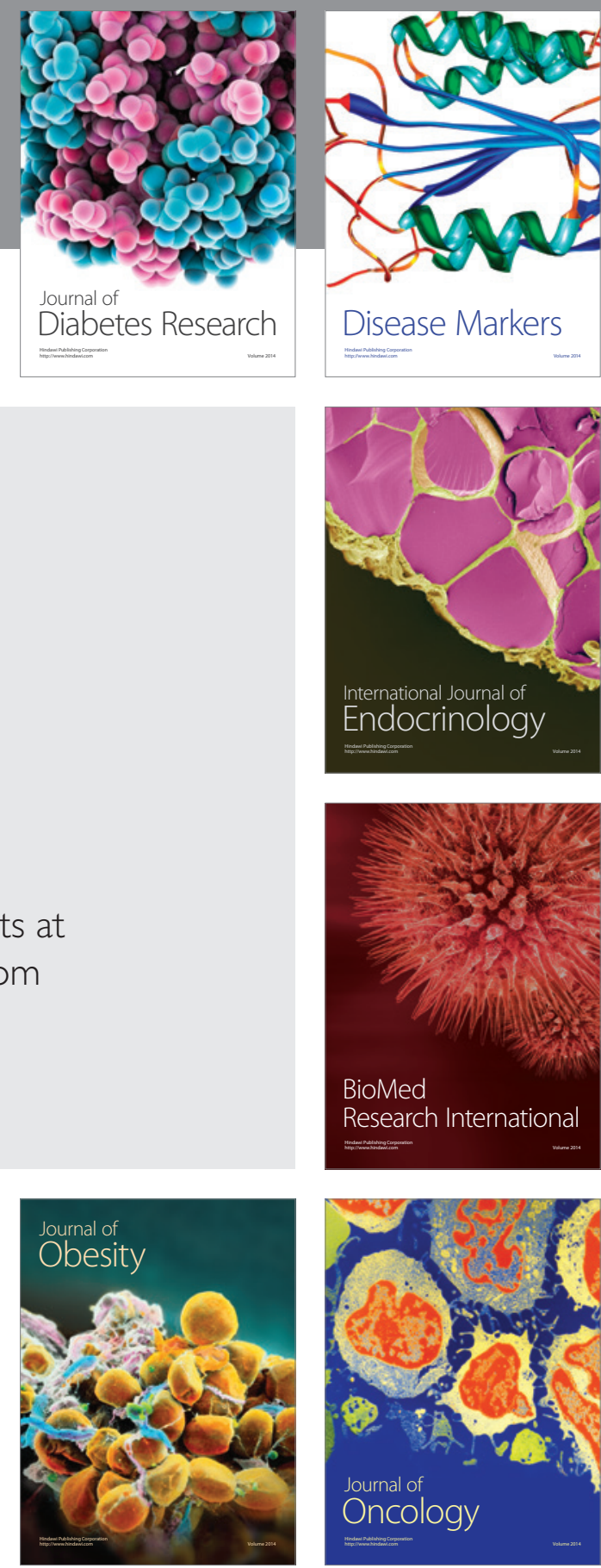

Disease Markers
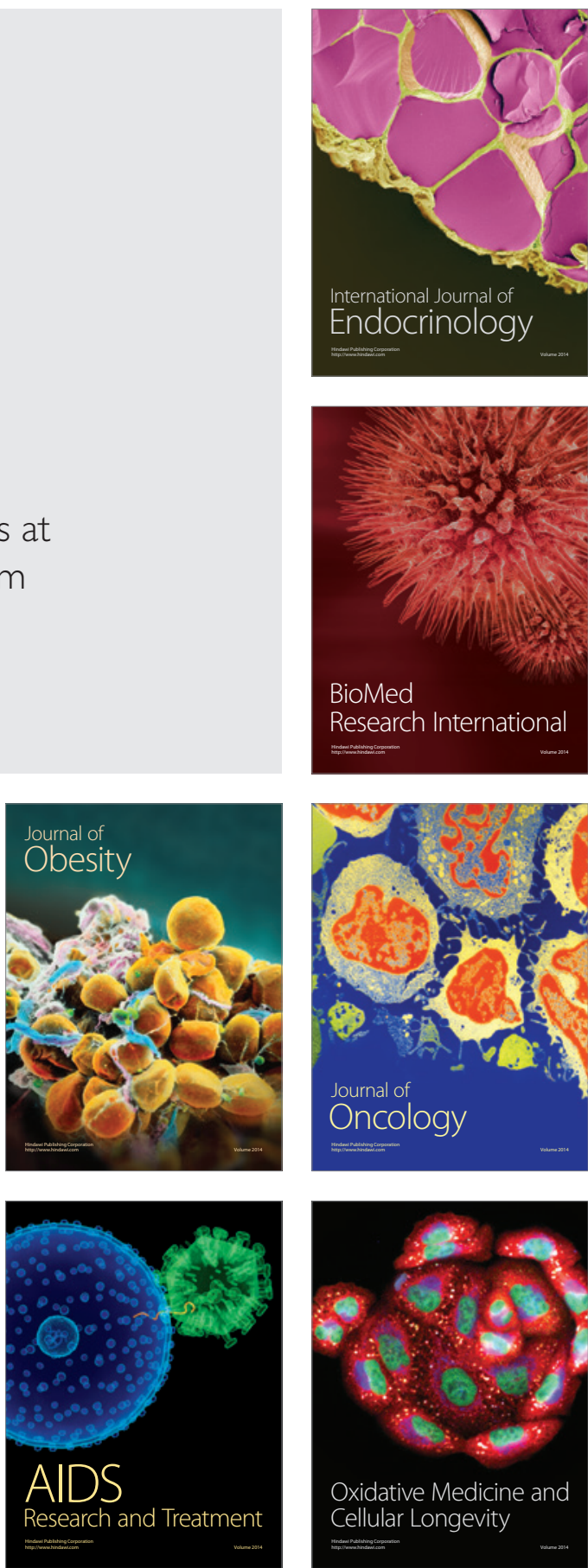